\title{
INHERENT VULNERABILITY OF FORESTS: A CASE STUDY FROM JHARKHAND IN INDIA
}

\author{
Langlentombi $\mathbf{L C}^{1, *} \&$ Kumar $\mathrm{M}^{2}$ \\ ${ }^{1}$ ICAR-Indian Institute of Natural Resins and Gums, Namkum-834010, Ranchi, Jharkhand, India \\ ${ }^{2}$ Forest Research Institute, PO: New Forest, Dehradun -248006, Dehradun, India \\ *lleishahen@gmail.com
}

Submitted September 2020; accepted December 2020

\begin{abstract}
Vulnerability assessment of forests is essential for identifying the degree of associated risk under present and future scenario to prioritize management interventions. The specific objective of the present study is to assess the vulnerability of natural forests in Jharkhand, India under the current situation. The natural forests face multiple stresses in the present state can be prone to additional stress of climate change. We assessed the vulnerability of forests under the prevailing current situation using indicators. The assessment involves the use of four indicators such as biological richness, canopy cover density, disturbance index and slope. A multi-criteria approach was adopted to assign weights to each of the indicators which were integrated in a GIS environment to map the spatial extent of the vulnerability. The entire study area was first divided into 787 grids of size $2.5^{\prime} \times 2.5^{\prime}$ to represent spatial extent of each indicators in the grids. The assessment indicated that only $2.92 \%$ forest grids fell under very high vulnerability class, whereas $18.68 \%$ grids came under high vulnerability, $53.88 \%$ were under medium and $24.52 \%$ grids were under low vulnerability classes. Very high vulnerability category of forested grids was largely located in Dry Peninsular Sal forests that required immediate management intervention.
\end{abstract}

Keyword: Vulnerability, indicators, forest management, climate change, multi-criteria analysis

\section{INTRODUCTION}

Vulnerability assessment of forests is essential to prioritize the management interventions and allocation of resources to protect the forests. Vulnerable forests in the present prevailing scenario may face the additional stress of climate change in due course and thus the assessment is essential to identify such forest types and their spatial extent. The vulnerability of a system can be defined as the susceptibility to disturbances determined by exposure to perturbations, sensitivity to perturbations and the capacity to adapt (Nelson et al. 2010). The vulnerability of a system is extensively referred in climate change literature to denote the extent of damage a region is predicted to suffer due to climate change (Deb et al. 2018). The vulnerability assessment is usually context specific. The present study on vulnerability in the context of the present prevailing situation and not to include the assessment for future projected scenarios of climate change. Forest ecosystems play a big role in stabilizing global climate change while they also regulate climate at the regional and global scale. Since forests are important carbon sinks and sources, assessing forest health and the extent of vulnerability of forests has gain much attention in recent years (Murthy et al. 2011). The vulnerability of forests was assessed by identifying prominent measurable factors for the quantification and mapping of the spatial extent of vulnerability which was referred as indicators for vulnerability.

The assessment of forested landscapes vulnerability requires comprehensive understanding of the factors that affect the forests and its attributes (Kumar et al. 2020a). The assessment of vulnerability of forest and agriculture ecosystem for future scenarios are usually done using simulation models (Kalra \& Kumar 2019, Kumar et al. 2018a). The assessment of vulnerability for the present study adopted the indicator-based simple approach. (Kumar et al. 2018b, Olokeogun \& Kumar 2020, Pokhriyal et al. 2020). Dominant forest treats such as fire, human pressure, cattle grazing, slope and soil related attributes or the internal state of the forest such as biodiversity, canopy density and productivity can be used as indicators of vulnerability. However, the selection of indicators is usually dependent upon the capability to measure the spatial extent of these indicators. Over the recent decades with the 
development and application of multiple sensors used in remote sensing, availability of measured values of multiple indicators has improved significantly. The developments have paved the way to facilitate the use of such variables as indicators for mapping vulnerability for different landscapes including forests. The present study used a simple approach to integrate selected indicators to map vulnerability of a forested landscape.

The vulnerability assessment of forests was referred to as inherent vulnerability and defined as the vulnerability of a system as a property that determines the capacity of a system to resist a disturbance and adjust to it (Sharma et al. 2013). The specific objectives of the study were to assess the inherent vulnerability of forests in Jharkhand under the present conditions and to identify dominant forest types falling under different vulnerability classes.

\section{MATERIALS AND METHODS}

\section{Study area}

The study was implemented in the forested landscape of Jharkhand in India. It has a total geographical area of $79,710 \mathrm{~km}^{2}$. The study area lies between $21^{\circ} 58^{\prime}-25^{\circ} 08^{\prime} \mathrm{N}$ and $83^{\circ} 19^{\prime}-87^{\circ}$ $55^{\prime} \mathrm{E}$ (Figure 1). The study area is surrounded by the state of West Bengal on the east, Chhattisgarh on the west, Bihar on the north and Orissa on the south. Vast areas of Jharkhand lie on the Chota Nagpur Plateau. The local climate varies from humid subtropical in the north to tropical wet and dry in the southeast. The southwest monsoon from mid-June to October brings $1000 \mathrm{~mm}$ of rainfall in the west-central part of the state to more than $1500 \mathrm{~mm}$ in the southwest.

\section{Data used}

Open access digital elevation data of Shuttle Radar Topography Mission at $30 \mathrm{~m}$ was downloaded from the USGS Earth Explorer website (USGS 2019). The Shuttle Radar Topography Mission data was used to generate slope map for one of the indicators of vulnerability. Two important layers developed by Indian Institute of Remote Sensing under the National Biodiversity Characterisation at Landscape project were used for biological richness index and disturbance index as indicators of vulnerability. Data of these two indices were downloaded from the Indian

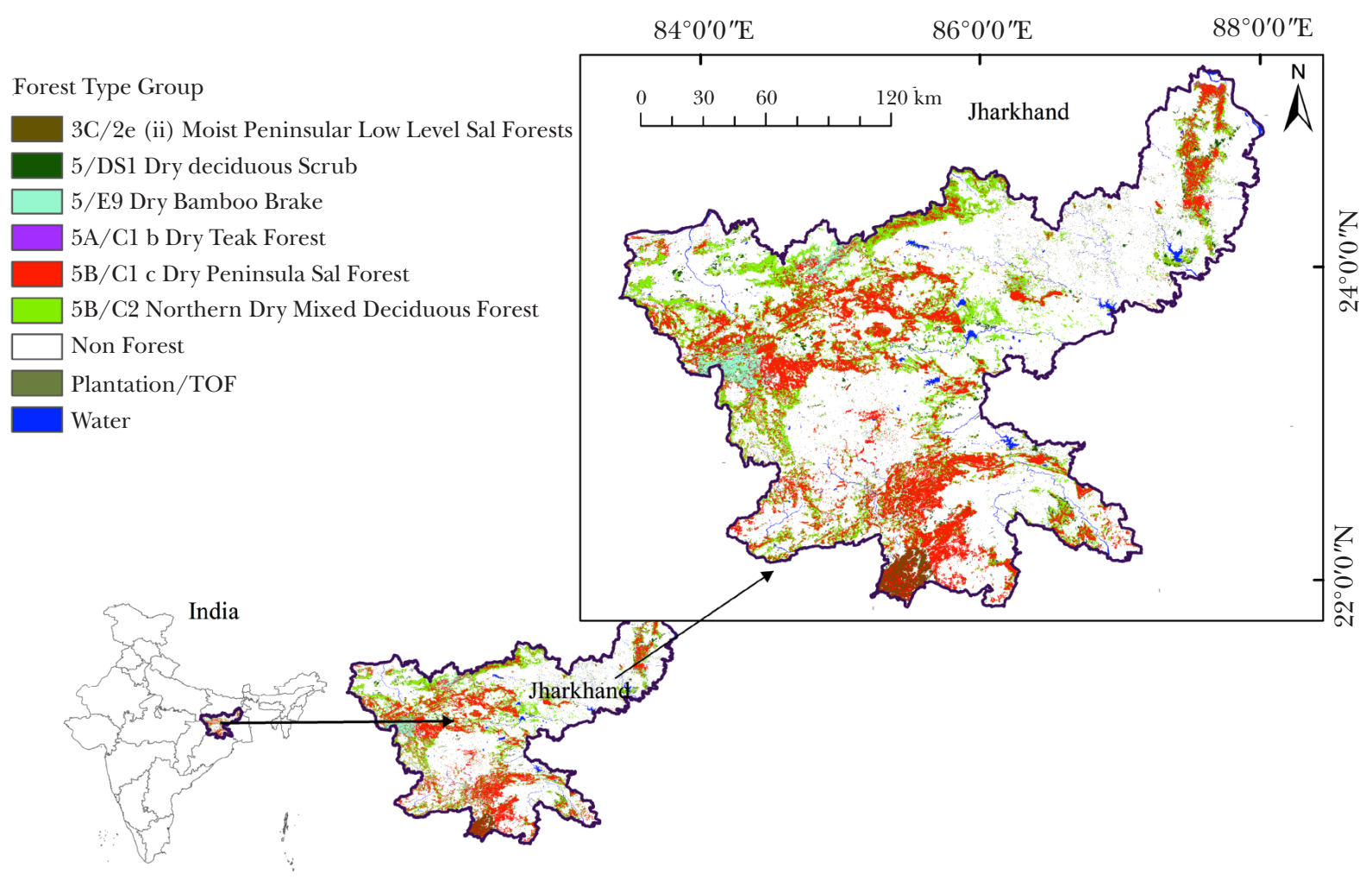

Figure 1 Jharkhand state of India and its dominant forest types 
Institute of Remote Sensing website (IIRS 2019). Forest cover density and the forest type of the study region were procured from the Forest Survey of India (FSI 2019). Forest cover density layer was used as one of the indicator layers while forest type layer was used to visualise final vulnerability classes for each of the forest types.

\section{Spatial analysis}

The four indicator layers of vulnerability such as slope, biological richness index, disturbance index and forest cover density were clipped for the study region and maintained the common pixel size of $30 \mathrm{~m}$ for all of the four layers in a raster format. Spatial analyst tool of ArcGIS 10.3 was used to calculate vulnerability at pixel level for the raster-based analysis.

\section{Vulnerability analysis}

Methodology for the assessment of vulnerability methodology was divided into four steps.

\section{Step 1: Identification of Indicators}

Prominent indicators linked to the vulnerability were identified by literature survey and discussion with the forest managers and experts. A given landscape may have multiple indicators, only four indicators (Table 1) were chosen for the study. The four indicators largely influenced the vulnerability of forests of the study region with measured values and were accessible for use as raster layer.

\section{Step 2: Weight assignment to indicators}

Multi criteria analysis and pairwise comparison method were used of to develop weights for the indicators (Wang et al. 2008). The study also took into consideration the opinion of ten experts to assign relative weights to each of the indicators to obtain final weights as suggested by Saaty (2008).

\section{Step 3: Integration of indicator layers using GIS}

Raster calculator available under Spatial Analyst tool of ArcGIS 10.3 was used to integrate all four indicator layers to calculate final vulnerability at a pixel resolution of $30 \mathrm{~m}$. Each of the indicator layers was first categorized into low, medium, high and very high classes as indicated in Table 1. Each of these classes for all four indicator layers was assigned values from 1 to 4 , where 1 indicated low vulnerability while 2, 3 and 4 showed higher values of vulnerability in increasing order. Spatial variation of vulnerability was also assigned within each layer while each of these layers was separately assigned individual weights following the methodology suggested in Step 2.

Table 1 Selected indicators with range of values for vulnerability classes and weights assigned to different vulnerability classes

\begin{tabular}{|c|c|c|c|}
\hline Indicators & Range of indicator value & Vulnerability class & Weight assigned \\
\hline \multirow[t]{4}{*}{ Biological richness index } & $2-33$ & (very high) & (4) \\
\hline & $34-49$ & (high) & (3) \\
\hline & $50-69$ & (medium) & $(2)$ \\
\hline & $70-90$ & (low) & (1) \\
\hline \multirow[t]{4}{*}{ Disturbance index } & $11-18$ & (low) & (1) \\
\hline & $19-24$ & (medium) & (2) \\
\hline & $25-28$ & (high) & (3) \\
\hline & 29-90 & (very high) & (4) \\
\hline \multirow[t]{4}{*}{ Canopy cover density (\%) } & $>70 \%$ & (low) & (1) \\
\hline & $40-70 \%$ & (medium) & (2) \\
\hline & $10-40 \%$ & (high) & (3) \\
\hline & $<10 \%$ & (very high) & (4) \\
\hline \multirow[t]{4}{*}{ Slope $\left({ }^{\circ}\right)$} & $<4^{\circ}$ & (low) & (1) \\
\hline & $4-10^{\circ}$ & (medium) & (2) \\
\hline & $10-19^{\circ}$ & (high) & (3) \\
\hline & $>19^{\circ}$ & (very high) & (4) \\
\hline
\end{tabular}


Step 4: Developing an inherent vulnerability profile

The final integrated value of vulnerability obtained at Step 3 was further classified into four different classes of vulnerability such as low, medium, high, and very high using Jenks Natural Breaks algorithm using ArcGIS 10.3. The final assessment of vulnerability for different forest types was done by evaluating the proportion of different vulnerability class pixels representing each forest types (Figure 2).

\section{RESULTS AND DISCUSSIONS}

The forest area of different forest type in order from high to low percentage was dry peninsular Sal forest, northern dry mixed deciduous forest, dry Bamboo brake, moist peninsular low level Sal forest and dry deciduous scrub respectively (Table 2). Out of the 787 forest grids in the landscape, the highest area $(55.78 \%)$ was occupied by the dry peninsular Sal forest while the dry deciduous scrub $(0.13 \%)$ represented the least percent of coverage. The assessment indicated that $53.88 \%$ forest grids fall under medium vulnerability followed by $24.52 \%$ as low and $18.68 \%$ under high vulnerability while only $2.92 \%$ forest grids showed very high inherent vulnerability.

Among all forest types, only dry peninsular Sal forest and northern dry mixed deciduous forest had very high vulnerable grids constituting 3.64 and $2.67 \%$ respectively (Table 3 ). Moist peninsular low level Sal forest was observed to be least vulnerable as compared to other forests. The higher inherent vulnerability of dry peninsular Sal forest could be due to majority of the area encompassed steep terrains. The study also observed that these regions faced high extraction by human activities making it more vulnerable.

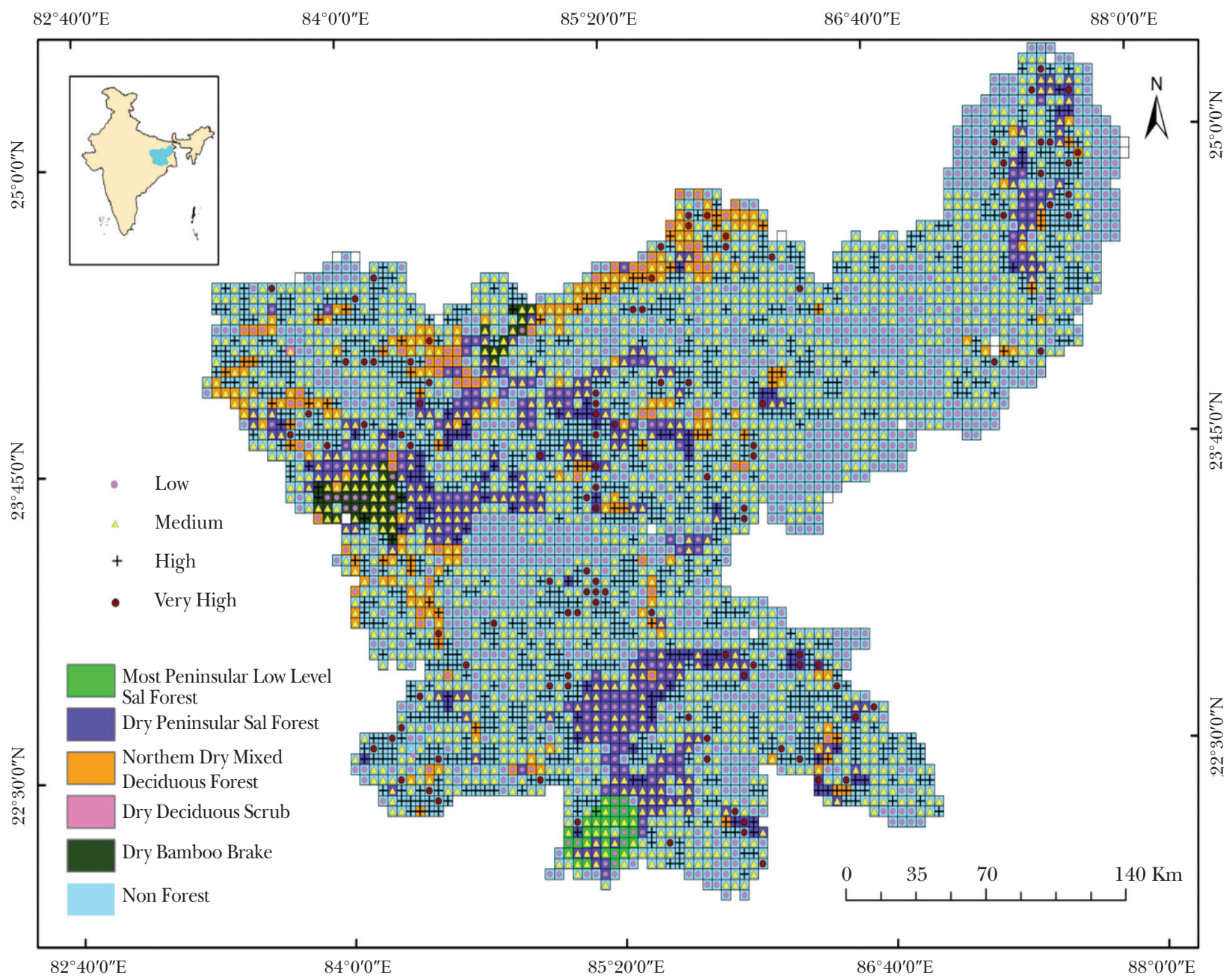

Figure 2 Spatial variation of inherent vulnerability classes (low, medium, high and very high) in different forest types 
Table 2 Forest types, forest area and forest grids of the study area

\begin{tabular}{lccc}
\hline Forest types & $\begin{array}{c}\text { Forest area } \\
\text { (ha) }\end{array}$ & Number & Percent $(\%)$ \\
\cline { 3 - 4 } Moist Peninsular Low Level Sal Forest & $58,954.09$ & 33 & 4.19 \\
Dry Peninsular Sal Forest & $1,000,142.00$ & 439 & 55.78 \\
Northern Dry Mixed Deciduous Forest & $910,550.00$ & 262 & 33.29 \\
Dry Deciduous Scrub & $66,144.48$ & 1 & 0.13 \\
Dry Bamboo Brake & $89,268.68$ & 52 & 6.61 \\
\hline Total & $2,125,059.00$ & 787 & \\
\hline
\end{tabular}

Table 3 Forest types and percentage of total forest grids under different inherent vulnerability classes

\begin{tabular}{lcccc}
\hline Forest types & \multicolumn{3}{c}{ Inherent vulnerability (\% grids) } \\
\cline { 2 - 5 } & Low & Medium & High & Very high \\
\hline Moist Peninsular Low Level Sal Forest & 33.33 & 63.64 & 3.03 & 0 \\
Dry Peninsular Sal Forest & 25.74 & 52.16 & 18.45 & 3.64 \\
Northern Dry Mixed Deciduous Forest & 22.14 & 52.67 & 22.52 & 2.67 \\
Dry Deciduous Scrub & 0 & 100.00 & 0 & 0 \\
Dry Bamboo Brake & 21.15 & 67.31 & 11.54 & 0 \\
\hline
\end{tabular}

Although the extraction pressure was not considered for assessing the vulnerability, it could be considered as one of the important drivers of vulnerability while assessing vulnerability of the forest. The vulnerability assessment of forests in the East Bokaro district, Jharkhand was done by Upgupta \& Singh (2017) using indicators to map composite vulnerability index values. They observed that more than $90 \%$ of the forested grids were highly vulnerable due to coal mining. It implied that selection of indicators for mapping vulnerability would depend on site and situation. Srivastava \& Garg (2013) assessed the vulnerability of forests in India based on fire as the prominent factor and reported regional variation of fire related vulnerability. The magnitude of fire vulnerability was found to vary over different forested grids of India. Kumar et al. (2020a) assessed the vulnerability of riparian zones using indicators that were primarily prominent in the riparian zones of Uttarakhand in India. The same site would reflect different vulnerability once the assessment was done using different sets of indicators. Therefore, the selection of indicators was the most significant component for all studies.

The study attempted to demonstrate an approach for mapping spatial vulnerability by using simple indicators that were readily available for the study region. The assessment could further be improved by considering more appropriate indicators once the values of the selected indicators were available. There was also a need to assess the role of various indicators for their effect on spatial variation of vulnerability at different scale. Sensitivity analysis of selected indicators that might have different influence and relationship with vulnerability and the assessment of impacts of scale on the spatial extent of vulnerability could be undertaken for such studies.

Identification of appropriate indicators and assigning weights to each indicator after logical inference was the most important step for mapping vulnerability using the approach demonstrated by the study. The vulnerability analysis was done by selecting indicators for which spatial layers were available for its integration in a GIS environment. However, the study could further be improved once other indicators such as the intensity of forest resource extraction by the human (Kumar et al. 2020b), fire intensity (Srivastava \& Garg 2013) and deposition of ash on plants due to mining activity (Upgupta \& Singh 2017) were considered. 


\section{CONCLUSIONS}

Assessment of vulnerability at the regional level is of paramount importance to the forest managers in decision making to prioritise the allocation of resources for forest protection. Such assessment assists in identifying forest areas which are immediately vulnerable and are at risk of loss and degradation due to prevailing stressors. The present study had identified dry peninsular Sal forest and northern dry mixed deciduous forest had larger percentage of grids under high and very high vulnerability classes. Therefore, these forests were considered for immediate intervention in their conservation and protection. This case study was expected to help forest managers and policymakers to address the risks associated with forests at the regional level while similar approach for other regions could be adopted after considering the most influential indicators which governed the vulnerability of the landscape.

\section{ACKNOWLEDGEMENTS}

The authors thanked the GIS Centre of Forest Research Institute, Dehradun for providing necessary facilities and the laboratory members of the GIS centre for their kind assistance during this study.

\section{REFERENCES}

Deb JC, Phinn S, Butt N et al 2018. Climate change impacts on tropical forests: Identifying risks for tropical Asia. Journal of Tropical Forest Science 30: 182-194. https:/ / doi.org/10.26525/jtfs2018.30.2.182194

FSI. 2019. Forest Survey of India, India State of Forest Report, Government of India, Dehradun.

IIRS 2019, Indian Institute of Remote Sensing: National Biodiversity Characterisation at Landscape Project. https://bis.iirs.gov.in/

Kalra N \& Kumar M. 2019. Simulating the impact of climate change and its variability on agriculture $\mathrm{Pp}$ 21-28 in Sheraz Mahdi, S. (Ed), Climate Change and Agriculture in India: Impact and Adaptation. Springer International Publishing, Cham. https://doi. org/10.1007/978-3-319-90086-5_3

Kumar M, Kalra N \& Ravindranath NH. 2020a. Assessing the response of forests to environmental variables using a dynamic global vegetation model: an Indian perspective. Current Science. 118: 700-701.
Kumar M, Rawat SPS, Singh H et al. 2018a. Dynamic forest vegetation models for predicting impacts of climate change on forests: An Indian perspective. Indian Journal of Forestry. 41: 1-12.

Kumar M, Savita \& Kushwaha S. 2020b. Managing the forest fringes of India: A national perspective for meeting sustainable development goals Pp 331-347 in Khaiter P \& Erechtchoukova M (eds) Sustainability Perspectives: Science, Policy and Practice. Springer Nature, Switzerland.

Kumar M, Singh H, Pandey R et al. 2018b. Assessing vulnerability of forest ecosystem in the Indian Western Himalayan region using trends of net primary productivity. Biodiversity $\mathcal{E}^{\circ}$. Conservation. 28: 2163-2182. https://doi.org/10.1007/s10531018-1663-2

Murthy IK, Timari R \& Ravindranath NH. 2011. Climate change and forests in India: adaptation opportunities and challenges. Mitigation and Adaptation Strategies for Global Change 16: 161-175. https://doi.org/10.1007/ s11027-010-9261-y

Nelson R, Kokic P, Crimp S et al. 2010. The vulnerability of Australian rural communities to climate variability and change: Part II-Integrating impacts with adaptive capacity. Environmental Science $\mathcal{E}$ Policy 13: 18-27. https://doi.org/10.1016/j.envsci.2009.09.007

Olokeogun OS \& Kumar M. 2020. An indicator based approach for assessing the vulnerability of riparian ecosystem under the influence of urbanization in the Indian Himalayan city, Dehradun. Ecology Indicators. 119. https://doi.org/10.1016/j.ecolind.2020.106796

Pokhriyal P, Rehman S, Krishna GA et al. 2020. Assessing forest cover vulnerability in Uttarakhand, India using analytical hierarchy process. Modelling Earth System $\mathcal{E}$ Environment. 6: 821-831 https://doi.org/10.1007/ s40808-019-00710-y

SAATY TL. 2008. Decision making with the analytic hierarchy process. International Journal of Services Sciences 1: 83-98.

Sharma J, Chaturvedi RK, Bala G et AL. 2013. Challenges in vulnerability assessment of forests under climate change. Carbon Management, 4: 403-411. https://doi. org $/ 10.4155 / \mathrm{cmt} .13 .35$

SRIVASTAVA P \& Garg A. 2013. Forest fires in India: Regional and temporal analyses. Journal of Tropical Forest Science 25: 228-239.

Upgupta S \& Singh PK. 2017. Forest risk and vulnerability assessment: A case study from East Bokaro coal mining area in India. International Journal of Environmental and Ecological Engineering 11: 966-974.

USGS 2019, United States Geological Survey Earth Explorer. https://earthexplorer.usgs.gov/

WANG XD, Zhong XH, Liu SZ ET AL. 2008. Regional assessment of environmental vulnerability in the Tibetan Plateau: Development and application of a new method. Journal of Arid Environments 72: 1929-1939. https://doi.org/10.1016/j.jaridenv.2008.06.005 